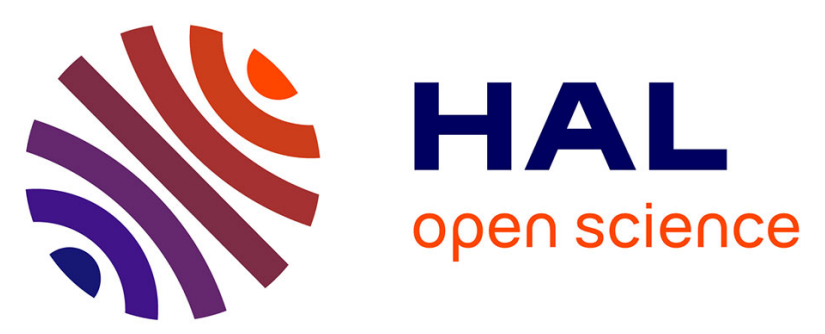

\title{
Building Modelling Methodology Combined to Robust Identification for the Temperature Prediction of a Thermal Zone in a Multi-zone Building
}

\author{
van Binh Dinh, Benoît Delinchant, Frédéric Wurtz, Hoang-Anh Dang
}

\section{To cite this version:}

van Binh Dinh, Benoît Delinchant, Frédéric Wurtz, Hoang-Anh Dang. Building Modelling Methodology Combined to Robust Identification for the Temperature Prediction of a Thermal Zone in a Multi-zone Building. The Sixth International Symposium on Integrated Uncertainty in Knowledge Modelling and Decision Making (IUKM 2018), Mar 2018, Hanoi, Vietnam. 10.1007/978-3-319-754291_19. hal-01738554

\author{
HAL Id: hal-01738554 \\ https://hal.science/hal-01738554
}

Submitted on 21 Apr 2020

HAL is a multi-disciplinary open access archive for the deposit and dissemination of scientific research documents, whether they are published or not. The documents may come from teaching and research institutions in France or abroad, or from public or private research centers.
L'archive ouverte pluridisciplinaire HAL, est destinée au dépôt et à la diffusion de documents scientifiques de niveau recherche, publiés ou non, émanant des établissements d'enseignement et de recherche français ou étrangers, des laboratoires publics ou privés. 


\title{
Building modelling methodology combined to robust identification for the temperature prediction of a thermal zone in a multi-zone building
}

\author{
Van-Binh Dinh ${ }^{1}$, Benoit Delinchant ${ }^{1}$, Frederic Wurtz ${ }^{1}$ and Hoang-Anh Dang ${ }^{2}$ \\ ${ }^{1}$ Univ. Grenoble Alpes, CNRS, Grenoble INP, G2Elab, F-38000 Grenoble, France \\ ${ }^{2}$ Hanoi University of Industry, Hanoi, Vietnam
}

\begin{abstract}
Building thermal modelling plays an important role in managing the thermal comfort and the energy consumption of buildings. A major challenge for modellers is how to deal with uncertainty problems in order to have a robust model with an acceptable computational time for the improvement of predictive control. This paper presents a methodology which allows obtaining the good model of a controllable thermal zone able to adapt regularly to the measurements by a robust identification procedure. Its input data are achieved by the modelling simplification of adjacent zones under uncontrollable uncertainties. This method is applied for a multi-zone positive energy building in south of France to validate our approach.
\end{abstract}

Keywords: Energy in buildings, robust identification, predictive control, thermal envelope modelling.

\section{Introduction}

Faced with the climate change and the limit of fossil fuels, the modelling and controlling building energy consumption are essential because buildings use $40 \%$ of the world's primary energy [1]. Among the energy consumers in buildings, space heating and/or cooling systems are important consumers assuring the human thermal comfort.

The energy consumption of heating and air conditioning systems depends on not only the climate conditions but also thermal characteristics of building envelope and occupant behaviour. Indeed, a building is considered as a complex system made up of different entities like walls, energy systems, and occupants under the external conditions such as weather conditions, sun or wind. The internal volume of the building and the external environment are separated by the surface of walls, also called building envelope. It is through this envelope that thermal exchanges operate. The characteristics of the envelope as well as the thermal interaction around it directly influence the inside air temperature and also the consumption of heating and cooling systems. Therefore, having a reliable building thermal envelope model performing well the simulated results compared to the measured ones, becomes an important mission for the model-based predictive control and the building energy management. However, 
due to the complexity of the built environment and uncertainty problems, it is not easy to achieve an accurate representation of real-world building operation.

The sources of uncertainty in building thermal modelling are generally associated to the inputs including dynamic and static types. It can be distinguished by 3 sources of uncertainty in building's operating phase:

- Uncertainties arising from static parameters as materials and dimensions of building envelope, window characteristics, infiltration rate etc. These uncertainties are due to the lack of knowledge about physical and geometrical parameters, defects during construction, or the aging of materials etc.

- Uncertainties related to dynamic parameters or solicitation variables such as weather data and occupant scenarios. Indeed, the measurement sensors of temperature, solar irradiation, and wind speed are not always available so that one does not sufficiently have input data for modelling. In addition, it is hard to estimate the number of people in a room, their behaviour and equipment use (turn on/off an air conditioning, open/close windows...). The prediction of these data for the next day is even far more difficult to obtain with good confidence.

- Uncertainties due to the modelling assumptions. It's about the simplification of building geometry or the limit of number of thermal zones for instance without taking into account the major effects that these approximations can induce.

As a consequence, a calibration can be done in order to match the simulation outputs with measured data, and then compensate all these uncertainties. But in prediction, it is important to keep the physical meaning of model parameters which is a trade-off to find with calibration.

Amara et al. [2] introduced building modelling approaches and devised them into three categories: white-box, black-box and grey-box. The white-box approach (sometimes called knowledge modelling) is based on the physical laws to describe the set of phenomena of building. This approach allows extrapolating the models to other situations which have been not present previously. Nonetheless, it requires a significant amount of building knowledge which is not compatible with real uncertainties. While the black-box models (or universal models), such as polynomial models (ARX, ARMAX...), are built from the observations without using a priori physical knowledge. It is very hard to extrapolate these models for the accurate prediction while the quality of these models strongly depends on the training data used as input. Finally, the authors indicated that the grey-box approach (semi-physical approach), based on both physical knowledge and observations, is the best alternative for the predictive control. By getting all strengthen from white-box and black-box approaches, the final approach permits to obtain lightweight models which can be rapidly tuned in conserving the physical meaning. The equivalent electrical circuit [3] using the thermal-electrical analogy is well known as a grey-box approach for the building thermal model.

The main purpose of this paper is to propose a modelling methodology using the equivalent electrical circuit to control a thermal zone in a multi-zone building. Adjacent zones, that one has very little information about, and that cannot be controlled, will be simplified. The controllable thermal zone model is then regularly calibrated by 
an automatic and robust identification procedure using our meta-optimization approach based on a scatting analysis.

The remainder of the paper is organized as follows: Section 2 reviews the methods and works associated to uncertainties for the building energy models. Then, our methodology is detailed and applied to positive energy building in section 3. Section 4 summarizes the main conclusions of the paper and proposes some future works.

\section{A brief overview of methods and works associated to uncertainties for building energy models}

\subsection{Sensitivity analysis}

The sensitivity analysis is the technique studying the effect of input parameters $x$ on the uncertainty of the output $y=f(x)=f\left(x_{1}, x_{2}, \ldots, x_{i}, \ldots, x_{n}\right)$. The technique allows to: (i) identify the input parameters having a significant or negligible influence on the output; (ii) determine the interactions between parameters, permitting a concentration on a group of parameters rather than separated parameters.

Saltelli et al. [4] has given a broad overview on methods of sensitivity analysis applied in many fields. In our article, popular methods used in the building science are briefly presented.

\section{Screening methods}

Screening methods are generally used for the computationally expensive models with a large number of inputs. It is based on the "One At a Time" OAT design which modifies one by one each input in fixing others and recording the results. In particular, the method of Morris [5] discretizes the space of input factors and constructs a series of trajectories in this space in moving inputs randomly one-parameter-at-a-time. This method permits to classify the inputs into 3 groups according to their effects: inputs having negligible effects, inputs having linear effects without interactions, and ones having nonlinear effects and/or interactions.

As a result, the screening methods allow obtaining qualitative information on the non-influential and influential inputs with a limited number of simulations but they do not quantify exactly the relative importance of a parameter compared to another, as well as the interaction between them. An application example of these methods was given by Heiselberg in [6] that measured the influence of design parameters on total energy demand of an office building in Denmark.

\section{Local sensitivity methods}

Based on the OAT approach too, local sensitivity methods evaluate the output variability in terms of the variation of one parameter at a time around its reference value, while all other parameters are held constant. In this case, a sensitivity index estimates the partial derivative of the output $y$ with respect to the parameter $x_{i}$ in the neighbourhood of its nominal value:

$$
S_{i}=x_{i} * \frac{d y}{d x_{i}}
$$


Local sensitivity methods require a reasonable number of simulations to obtain the quantitative information for the analysis of the influence of inputs on the output of the models with an important number of parameters. Nevertheless, these methods only take into account the local effect of input parameters but neither their variation range nor the correlation between them. Westphal and Lamberts [7] used the local sensitivity analysis to specify thermal loads for the calibration of a public office building. Mejri [8] applied this method for the identification of dynamic models for the performance evaluation and energy diagnosis of existing buildings. It has also applied to illustrate the importance of occupancy and indoor temperature of the residential building in Czech Republic [9].

\section{Global sensitivity methods}

Global sensitivity methods help studying the influence of input parameters in their all range of variation. This approach takes into account the probability distribution of each input and in many cases all parameters can be simultaneously varied for observing the interactions between them. According to Lavin et al. [10], global sensitivity analysis methods can be categorized into three groups: Monte Carlo based methods, variance based methods, and graphical methods.

Monte Carlo based methods aim at making a large number of evaluations with randomly selected model inputs, and then using regression-based measures [11-12] to analyse the contribution of input factors to the output uncertainty.

Variance based methods, such as Sobol [13, 14] and FAST [15, 16], study how the variance of output is due to the variation of each parameter and its related interactions via sensitivity indexes of different orders.

Graphical methods estimate the qualitative measures of sensitivity using the graphical assessment with charts, graphs, or surfaces of pairs of inputs-corresponding outputs. These methods can bring us the complementary visual information about the meaning of numerical sensitivity indices and the enhancement of results of other quantitative methods. Scatterplot [17] is one of the most common forms of graphical sensitivity methods.

\subsection{Model calibration}

Model calibration aims at tuning the model parameters so that simulation outputs match closely with the measured data.

According to Clarke et al. [18], calibration methodologies can be grouped into 4 principal categories: calibration based on manual, iterative and pragmatic intervention; calibration based on a suite of informative graphical comparative displays; calibration based on special tests and analytical procedures; and calibration based on analytical and mathematical methods. Reddy [19] revised these methods and agreed that the first three categories of the group involves tuning and refining the initial simulation input parameters in a heuristic manner, which depends on the experience and expertise of the user. On the contrary, the final one based the analytical and mathematical formulation is associated to an optimization problem which identifies automatically multiple solutions within a parameter space to minimize an objective func- 
tion. Reddy also indicated that sensitivity analysis should be used in the first stage of calibration process for reducing the number of parameters to be calibrated so that the numerical optimization is more efficient. Such a systematic and automated way is applied by O'Neill and Eisenhower [20] who used a sensitivity analysis for identifying the most important parameters for tuning an office building energy model. An overview of applications of the building model calibration can be found in [21, 22].

\subsection{Our proposals for the improvement of building thermal models under uncertainties}

In order to contribute to the uncertainty management in modelling, we have proposed some approaches for improving the reliability of building energy and thermal models.

Grandjacques et al. [23] developed sensitivity analysis methods for dependent and dynamic inputs based on the method of Pick and Freeze for the estimation of Sobol's indexes. These methods were applied for an existing platform building in France to take into account both the dynamic aspect of the inputs (room temperatures, heating system, presence of occupancy, inertia of heat exchange) and the one of the output.

Dang et al. [24] presented a thermal envelope model based on the equivalent electrical circuit for the building optimal management. From 16 input parameters including thermal resistances and capacitances, the method of Morris was used to determine 8 non-influential inputs which were then fixed by their analytic values. The rest of input factors which have a significant influence on the output were calibrated with one data set and then validated with only another data set. It is notable that such a model is not still robust since the model may provide poor performances when applied to environmental conditions that significantly differ from those which calibration referred to. That is why the model recalibration, and even the regular and automatic recalibration, becomes more and more important in real time predictive control. However, it is essential to understand that more parameters of model there are, less robust the calibration process is, because of the convergence problems in particular.

With the aim of improving the robustness of calibration for predictive control, we have developed in Nguyen Hong et al. [25] a calibration procedure of building energy models using a called meta-optimisation approach with the help of scattering parameters analysis. In this study, the scattering analysis aims to reach a reduced number of parameters to be identified. The parameter estimation is then performed by an automatic and regular optimization process to obtain the more robust prediction relative to the environment change. Through different tests, the authors also demonstrated the performance of this approach compared to the classical methods in terms of computational cost, prediction errors. Nevertheless, it is still a big challenge to apply this approach for real complex buildings with an important number of zones, such as office, commercial or apartment buildings. Indeed, modelling the overall system is a really great challenge, and the convergence issues of the optimization process applied to multi-zone buildings is still present.

In the context of studying the thermal model for predictive control of a zone in a multi-zone building, the uncertainties significantly increase when ones do not have any knowledge and information about the adjacent zones that directly influence the 
temperature of considered zone. Therefore, modelling whole building with the same level of detail for each zone is called into question. Considering our past studies, the next part is addressing the issue of robust calibration for the predictive control of a single zone in a multi-zone building.

\section{Building modelling methodology based on the robust identification for the temperature prediction of one thermal zone in the building}

\subsection{Methodology: assumptions for simplification}

Our methodology aims to improve the modelling efficiency permitting a more robust calibration process for the temperature prediction of one thermal zone located in a multi-zone building as described in the figure 1.

On the left of this figure, the 2-way arrows represent the 2-way interactions between the building zones in prediction. In order to predict the temperature of the studied zone, called "zone 1", it is necessary to know weather conditions and the temperatures of adjacent zones (2, 3 and 4). However, the temperatures of adjacent zones, themselves, are also unknown and have to be predicted depending on the temperature of "zone 1", and other boundaries. Consequently, the crossed interaction between building zones requires the model of whole building for the prediction of only one zone.

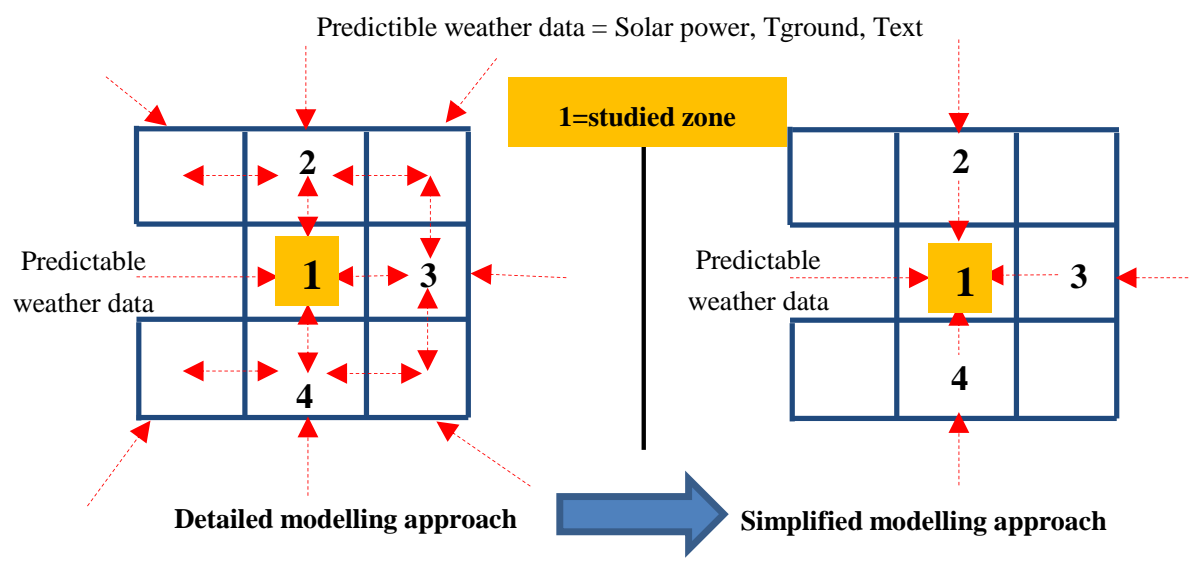

Fig. 1. Modelling assumptions for the temperature prediction of a studied zone "1"

As previously discussed, the grey-box model in the form of equivalent electrical circuit is a good choice for prediction. Nevertheless, using this semi-physical approach for modelling the whole building can bring a complex model with a large number of parameters which are difficult to calibrate regularly and robustly. Regarding the lack of information about adjacent zones (scenario of use, materials...), our methodology is as following: 
- simplify the modelling for adjacent zones by the assumption that their temperatures in prediction depend only on the exterior environment (such as solar power, exterior temperature and ground temperature which are predictable by weather forecast services) and neither on the zone of interest (zone 1) nor on other boundaries. This can be illustrated on the right of the figure 1 .

- use simple approximate models (simplified structure of an equivalent electrical circuit) to predict the temperatures of the adjacent zones and then take the results obtained as input data for the prediction model of zone 1.

\subsection{Methodology: electrical equivalent models for the zone of interest and adjacent zones}

Generically, the equivalent electrical circuit of zone 1 can be seen as figure 2. This circuit considers that electrical components like voltage sources, current sources, resistors and capacitors are respectively corresponding to temperatures, heat gains, thermal resistances and capacitances. The 2R1C-structure used for each wall (contact between 2 zones) is considered as a good trade-off between the accuracy and the robust prediction. Particularly, $\mathrm{R}_{- \text {ext_1}}, \mathrm{R}_{-\mathrm{ext} \_ \text {2 }}$ and $\mathrm{C}_{\text {_ext }}$ represent respectively the external resistance, internal resistance and capacitance of wall of zone 1 linked to exterior. $\mathrm{R}_{\_} \mathrm{adj}_{2} 1$ and $\mathrm{R}_{\_}$adj2_2 and $\mathrm{C}_{\text {_adj2 }}$ represent the external resistance, internal resistance and capacitance of wall of zone 1 linked to adjacent zone $2 . \mathrm{R}_{\text {vent }}$ introduces the resistance linked to the ventilation. The analytic values of resistances and capacitances are calculated from the envelope characteristics.

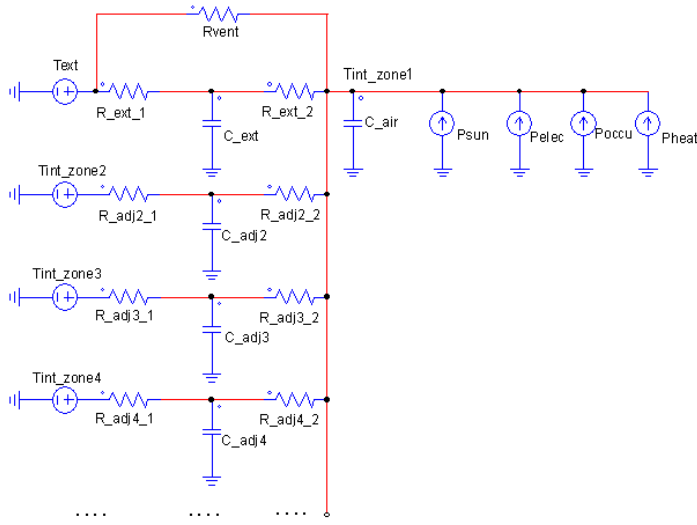

Fig. 2. Equivalent electrical circuit of zone 1

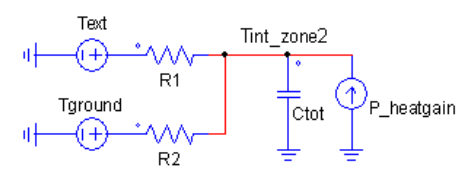

Fig. 3. Approximate model of adjacent zones (eg. zone 2)

$\mathrm{P}_{\text {sun }}, \mathrm{P}_{\text {elec }}, \mathrm{P}_{\text {occu }}$ and $\mathrm{P}_{\text {heat }}$ are heat gains inside the zone 1, which are associated to sun, electrical appliances, occupancy and heating system. $T_{\text {ext }}$ and $T_{\text {int_zone1 }}$ are external temperature and internal air temperature of zone 1. $\mathrm{T}_{\text {int_zone2 }}, \mathrm{T}_{\mathrm{int} \_ \text {zone3 }}$ and $\mathrm{T}_{\text {int_zone4 }}$ are respectively the air temperatures of adjacent zones. 
Meanwhile, with the proposed modelling assumptions, the temperature of adjacent zones, for example $\mathrm{T}_{\text {int_zone2 }}$ of zone 2 , is calculated by a much simpler circuit (Figure 3 ). Here only the heat exchange between zone 2 and exterior (and maybe zone 2 and ground if this zone is close to the ground) is taken into account by one resistance and one capacitance while the thermal effect of other boundaries on zone 2 is neglected.

The model of an adjacent zone (eg. zone 2) can be expressed by the equation:

$T_{\text {int_zone } 2}^{\prime}=\frac{1}{C_{\text {tot }}} *\left(\left(\frac{1}{R_{1}}+\frac{1}{R_{2}}\right) * T_{\text {int_zone } 2}+\frac{1}{R_{1}} * T_{\text {ext }}+\frac{1}{R_{2}} * T_{\text {ground }}+P_{\text {heatgain }}\right)$

It remains some physical meaning in parameters $R_{l}, R_{2}, C_{t o t}$, but less than those of the zone of interest. Thus, they can be obtained during calibration process from a simple temperature sensor or a simulation.

The simplification in modelling the adjacent zones is acceptable because the uncontrollable sources of uncertainty are very high so that a detailed modelling approach is not suited. Moreover, we are mainly interested in the behavior of zone 1 which will be frequently calibrated with measured data to fit with the environmental changes.

\subsection{Methodology: calibration}

A periodic recalibration process is using a meta-optimisation approach combined with scattering parameters analysis, as presented in Figure 4.

The first step aims at pre-training the model with the scattering parameters analysis to determine how many parameters among $n$ parameters of initial model should be fixed. The detailed description of this method can be seen in [25].

The step 2 is an automatic process during real time control, in which the scattering parameters analysis is one more time used with multi-start optimization to define which $k$ parameters have to be fixed in taking into account the data of first 8 days of the season. This is done each three-month to adapt to the season changes. This model is re-calibrated every two days to improve the prediction for the next days. Such a process is running continuously and automatically during one year. The application of this methodology is presented in the coming section, using the simplified model of a building based on adjacent zone approximations.

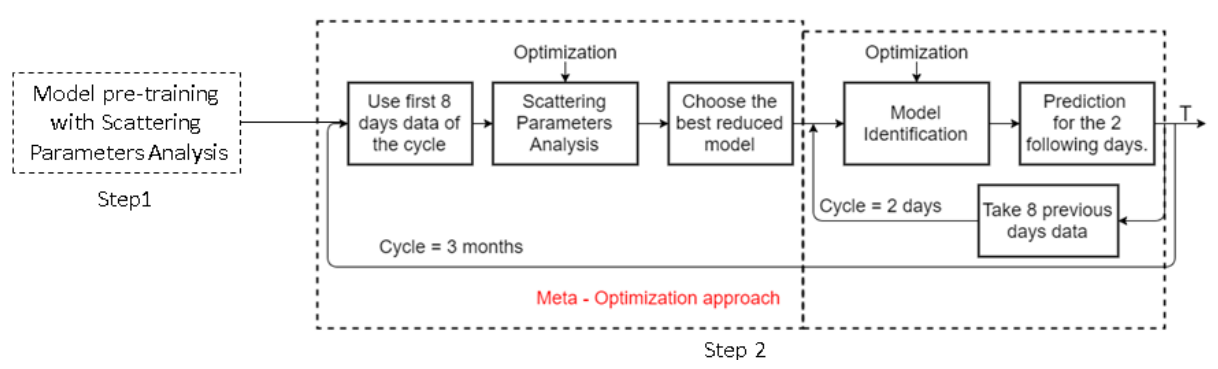

Fig. 4. Robust calibration procedure for temperature prediction of zone 1 


\subsection{Application for a positive energy building}

\section{Case study}

Our case study is a positive energy building located in south of France with a floor area of more than $200 \mathrm{~m}^{2}$. This household includes one main zone, called heated zone which is regulated by a heating system, one garage zone and two basements (room basement and office basement). It has been built, with high performance materials to reduce heat losses and to ensure a summer thermal comfort without cooling system.

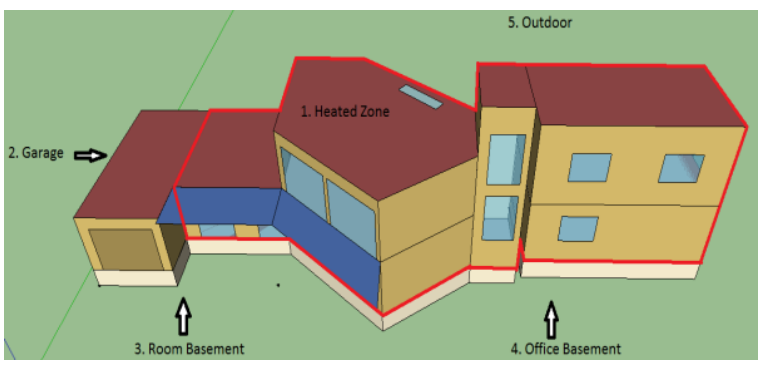

Fig. 5. Overview of studied building

The purpose of this study is to apply our methodology for the prediction of heated zone air temperature knowing that the garage and basement zones are under uncontrollable uncertainties because of different reasons (no sensors or limited sensors, uncontrolled scenarios of use...).

\section{Model of heated zone}

As proposed in our methodology above, an equivalent electrical circuit was constructed to produce the model for the heated zone. In this study, $T_{\text {int }}$, the inside temperature of heated zone, plays the role of $\mathrm{T}_{\text {int_zone1 }}$ in Figure 2. $\mathrm{T}_{\text {gar }}, \mathrm{T}_{\text {off }}$ and $\mathrm{T}_{\text {room }}$ are respectively the air temperatures of garage, office and room basement zones (adjacent zones of heated zone), playing the role of $\mathrm{T}_{\text {int_zone2 }}, \mathrm{T}_{\text {int_zone3 }}$ and $\mathrm{T}_{\text {int_zone4 }}$ in Figure 2.

\section{Model of adjacent zones}

The garage zone is thermally linked with outdoor, room basement and heated zone. As discussed in our methodology for modelling simplification, we neglect the thermal effect of heated zone on the garage zone and replace the heat transfer to the garage from the room basement by the ground in assuming that the thermal insulation between the room basement and the ground is so weak. In this case, the heat gain inside the garage comes mainly from the sun. As a result, we obtain the simplified model expressed by the equation:

$$
\begin{aligned}
& T_{\text {gar }}^{\prime}=\alpha * T_{\text {gar }}+\beta * T_{\text {ext }}+\gamma * T_{\text {ground }}+\delta * P_{\text {sun }} \\
& \text { With } \alpha=\frac{1}{C_{\text {tot }}} *\left(\frac{1}{R_{1}}+\frac{1}{R_{2}}\right) ; \beta=\frac{1}{C_{\text {tot }}} * \frac{1}{R_{1}} ; \gamma=\frac{1}{C_{\text {tot }}} * \frac{1}{R_{2}} ; \delta=\frac{1}{C_{\text {tot }}}
\end{aligned}
$$


The parameters $\alpha, \beta, \gamma$ and $\delta$ were identified in our study using a detailed dynamic thermal model (EnergyPlus ${ }^{1}$ software). It would have been done using measures. The prediction performance of our simplified model is evaluated using another dataset and shown in Figure 6 by a Bland Altman plot. It is observable that the simplified model is strongly correlated to data. The root mean squared error (RMSE) is $1.17^{\circ} \mathrm{C}$ while mean absolute error (MAE) is $1.08^{\circ} \mathrm{C}$. The coefficient of determination $\left(\mathrm{r}^{2}\right)$ is 0.9911 that is very close to 1 , expresses how well the predictions of our simplified model fits data.

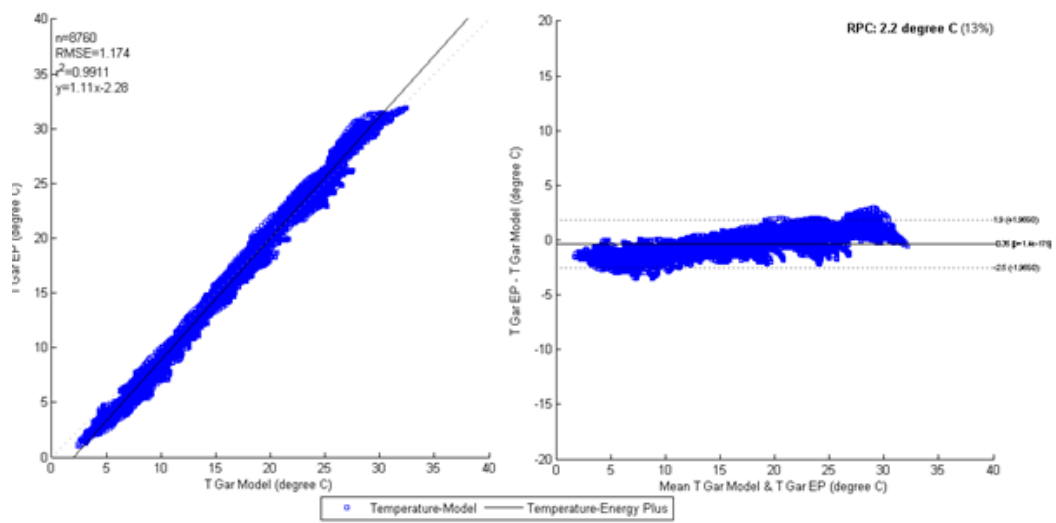

Fig. 6. Bland Atman plot between predictions of our simplified model and data.

Despite this, we are always aware that the temperature prediction of garage zone based on this simple model would be sometimes not accurate but the most important things are the prediction accuracy for the heated zone and the reduction of computation time.

A similar work was done for the modelling of office and room basement zones.

\section{Robust identification for heated zone temperature prediction}

We focus now on results achieved by applying our methodology with the robust calibration procedure mentioned in Figure 4 for the temperature prediction of heated zone. In fact, the pre-training in step 1 with scattering parameters analysis permitted to find the optimal degree of freedom for this model with 7 parameters to be fixed from 14 parameters of model. The automatic and continuous calibration process over one year in step 2 leads to very good predictions (Figure 7) with a mean error of $0.88^{\circ} \mathrm{C}$ and a maximal error of $2.51^{\circ} \mathrm{C}$.

\footnotetext{
${ }^{1}$ http://apps1.eere.energy.gov/buildings/energyplus
} 


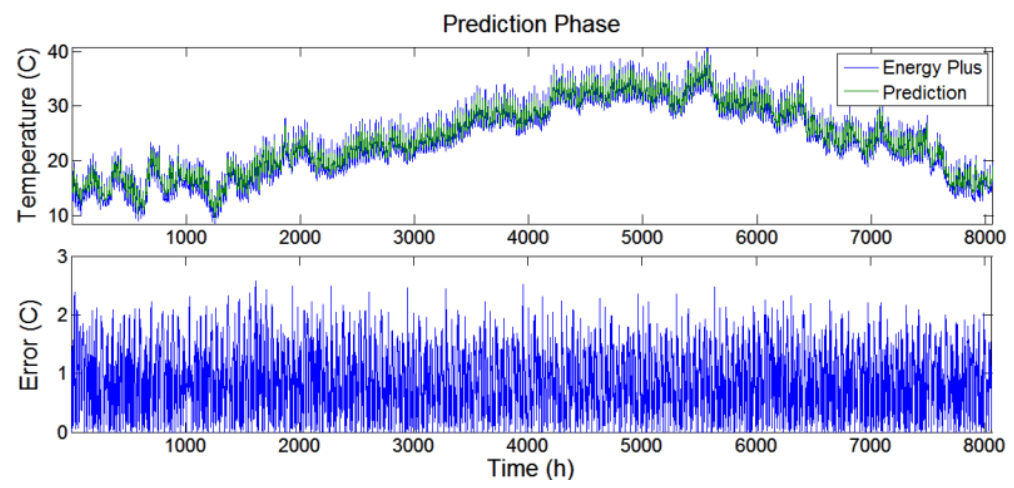

Fig. 7. Prediction results throughout one year

The prediction accuracy is very similar throughout the year, which can be explained by the regular and robust calibration process. The total computational time for whole year anticipation is about 1 hour 40 minutes with the time for pre-training model estimated of about 20 minutes.

\section{Conclusions and perspectives}

This paper has introduced a modelling methodology permitting to perform the robust identification to improve the prediction process of a thermal zone located in the multizone building. The obtained results indicate the robustness of the prediction with a reasonable computational time. It is to conclude that the simplification in modelling of adjacent zones with various uncontrollable uncertainties is a good solution to generate the data input for the temperature prediction of the studied main zone. Furthermore, this methodology should be tested for other cases study and be integrated into real time anticipative energy management system.

\section{References}

1. Costa, A., Keane, M.M.: Building operation and energy performance: Monitoring, analysis and optimisation toolkit. Applied Energy, vol. 101, 310-316 (2013).

2. Amara, F., Agbossou, K., Cardenas, A., Dubé, Y., Kelouwani, S.: Comparison and Simulation of Building Thermal Models for Effective Energy Management. In: Smart Grid and Renewable Energy, pp. 95-112 (2015).

3. Mendes, N., Oliveira, G.H., De Araújo, H.X.: Building thermal performance analysis by using matlab/Simulink. In: Seventh International IBPSA Conference, Rio de Janeiro, Brazil, pp. 473-480 (2001).

4. Saltelli, A., Ratto, M., Andres, T., Campolongo, F., Cariboni, J., Gatelli, D., Saisana, M., and Tarantola, S.: Global Sensitivity Analysis. The primer. John Wiley \& Sons, Ltd, 2008.

5. Morris, M.D.: Factorial sampling plans for preliminary computational experiments. Technometrics, 33, 161-174 (1991). 
6. Heiselberg, P., Brohus, H., Hesselholt, A., Rasmussen, H., Seinre, E., Thomas, S.: Application of sensitivity analysis in design of sustainable buildings. Renewable Energy, 34, 2030-2036 (2009).

7. Westphal, F. S., Lamberts, R.: Building simulation calibration using sensitivity analysis. In: $9^{\text {th }}$ IBPSA conference, Montréal, Canada (2005).

8. Mejri, O.: Développement de méthodes de diagnostic énergétique des bâtiments. $\mathrm{PhD}$ thesis, Universités de Tunis El Manar et Bordeaux 1 (2011).

9. Kominek, P., Tumova, E., Hirs, J.: Sensitivity analysis of residential building simulation parameters. In: $9^{\text {th }}$ International Conference, High Tatras, Slovakia (2017).

10. Lavin, R.B., Rohlig, K.J., Becker, D.K.: Sensitivity analysis techniques for the performance assessment of a radioactive waste repository. In: Euradwaste - Seventh European Commission Conference, ISBN: 978-92-79-13105-9, p. 387-397 (2008).

11. Hopfe, C.J., Hensen, J.L.M.: Uncertainty analysis in building performance simulation for design support. Energy and building, 43, 2798-2805 (2011).

12. Nguyen, A.T., \& Reiter, S.: A performance comparison of sensitivity analysis methods for building energy models. Building simulation, vol. 8, No 6, pp. 651-664 (2015).

13. Sobol, I.M.: Sensitivity estimates for nonlinear mathematical models. MMCE, vol. 1, No. 4, 407-414 (1993).

14. Sobol, I.M., Tarantola, S., Gatelli, D., Kucherenko, S.S., Mauntz, W.: Estimating the Approximation Error when Fixing Unessential Factors in GSA. Reliability and System Safety, Vol.92, pp.957-960 (2007)

15. Cukier, R.I., Fortuin, C.M., Shuler, K.E., Petschek, A.G. and Schaibly, J.K.: Study of the Sensitivity of Coupled Reaction systems to Uncertainties in Rate Coefficients. I. Theory. Journal of Chemical Physics, Vol. 59 (8), 3873-3878 (1973).

16. Saltelli, A., Tarantola, S. and Chan, K.: A Quantitative, Model Independent Method for Global Sensitivity Analysis of Model Output. Technometrics, Vol. 41, Number 1, 3956(1999.

17. Friendly, M., Denis, D.: The early origins and development of the scatterplot. Journal of the History of the Behavioral Sciences, 41 (2), 103-130 (2005).

18. Clarke, J.A., Strachan, P., Pernot, C.: An approach to the calibration of building energy simulation models. ASHRAE Trans., 99, 917-927 (1993).

19. Reddy, T.A: Literature review on calibration of building energy simulation programs: Uses, problems, procedures, uncertainty and tools. ASHRAE Trans., 112, 226-240 (2006).

20. O'Neill, Z., Eisenhower, B.: Leveraging the analysis of parametric uncertainty for building energy model calibration. Build. Simul., 6, 365-377 (2013).

21. Fabrizio, E., Monetti, V.: Methodologies and Advancements in the Calibration of Building Energy Models. Energies, 8, 2548-2574 (2015).

22. Coakley, D., Raftery, P., Keane, M.: A review of methods to match building energy simulation models to measured data. Renewable and Sustainable Energy Reviews 37, 123 - 141 (2014).

23. Grandjacques, M., Janon, A., Adrot, O., Delinchant, B.: Pick-freeze estimation of sensitivity indices for models with dependent causal processes inputs. MCQMC, KU Leuven, Belgium (2014).

24. Dang, H. A., Delinchant, B., and Wurtz, F.: Toward building energy management: Electric analog modeling for thermal behavior simulation. In: IEEE International Conference on Sustainable Energy Technologies (ICSET), Hanoi, pp. 246-250 (2016).

25. Nguyen Hong, Q., Le-Mounier, A., Dinh, V.B, Delinchant, B., Ploix, S., Wurtz, F.: MetaOptimization and Scattering Parameters Analysis for Improving On Site Building Model Identification for Optimal Operation. IPBSA, California, USA (2017). 